\title{
醛或酮脱氧对氮杂芳环碳氢键的直接烷基化
}

\author{
程骁恺陆 展* \\ (浙江大学化学系 杭州 310027)
}

\section{Ketones and Aldehydes as Alkyl Radical Equivalents for Direct C-H Alkylation of Heteroarenes}

\author{
Cheng, Xiaokai Lu, Zhan* \\ (Department of Chemistry, Zhejiang University, Hangzhou 310027)
}

氮杂芳环在天然产物、复杂分子等有机分子中广泛 存在, 因此对其合成及修饰具有非常重要的意义. 在酸 性和氧化条件下, 通过自由基加成的方式实现氮杂芳环 的碳-氢键烷基化的方法被称为 Minisci 反应. 这类反应 能够高效地合成烷基取代的氮杂芳环化合物, 然而高温 和需要过量的氧化剂及酸制约了该反应的适用范围. 目 前, Minisci 反应中自由基前体主要包括羧酸、烷烃、卤 代烃、硼酸、醇、胺及亚磺酸盐 ${ }^{[1]}$, 利用醛酮作为烷基 自由基前体参与 Minisci 反应的例子尚未见报道.

含羰基化合物(醛酮等)是非常重要的大宗化学品, 在天然产物的合成、材料、化工等领域中具有重要的地 位. 一直以来, 含羰基化合物(醛酮等)主要是作为亲电 试剂接受亲核试剂进攻参与有机化学反应的, 如 Aldol 反应和格氏试剂加成反应等 ${ }^{[2]}$. 而极性反转的策略 ${ }^{[3]}$ 能 够将原本亲电的羰基转化为亲核试剂, 为羰基化合物的 化学转化提供了更多选择. 醛也可发生氢原子转移反 应, 随后通过脱羰反应形成烷基自由基, 继而参与自由
基反应 ${ }^{[4]}$. 而酮羰基作为烷基自由基的来源, 参与自由 基反应的方法还没能够实现. 南开大学化学学院汪清民 课题组 ${ }^{[5]}$ 利用可见光促进的质子偶合电子转移(PCET) 在温和的条件下对醛酮进行单电子还原, 通过与生物体 中的自旋中心迁移(SCS)反应相结合，在温和的反应条 件下实现了以醛酮为烷基自由基源的 Minisci 反应.

反应的挑战性在于如何断裂碳氧双键，作者采取分 步走的策略，首先利用 Knowles 等发展的可见光促进的 质子偶合电子转移 ${ }^{[6]}$ 方法对醛酮进行活化, 首先完成了 $\pi$ 键的断裂, 将羰基转化为 $\alpha$-烷基自由基, 随后发生自 由基加成反应. 同时, 这一策略也解决了羰基化合物与 氮杂芳环的极性不匹配的问题. 在药物化学界, 鉴于烷 基对药物代谢和药代动力学的影响, 将其直接引入芳香 杂环的需求越来越大 ${ }^{[7]}$, 因此需要对反应过程中的加成 产物进行羟基脱除. 在生物体内核糖核苷在核糖核苷酸 还原酶的作用下发生 $\beta$-碳氧键自由基断裂脱除羟基形
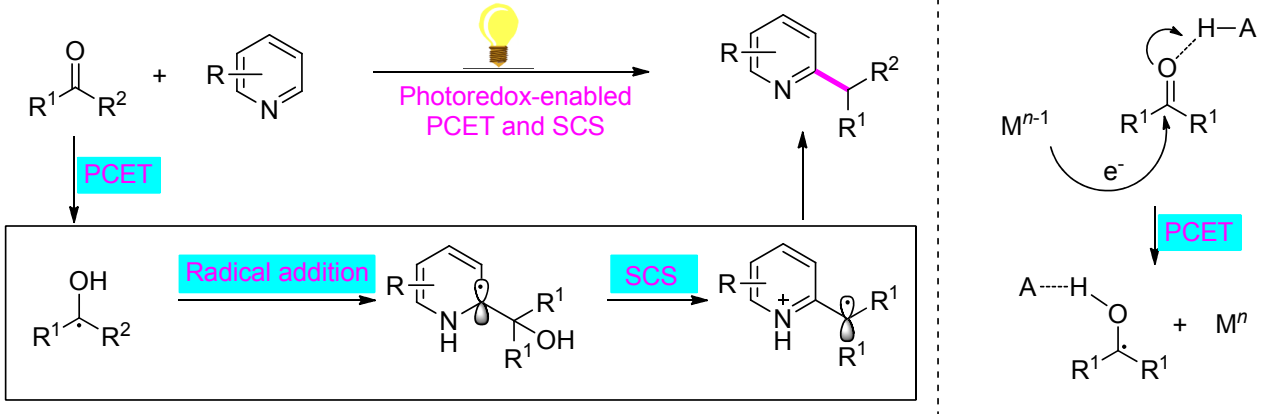

* Corresponding author. E-mail: luzhan@zju.edu.cn. published online November 11, 2019. 
成脱氧核苷. 借用这一策略通过 SCS 过程 ${ }^{[8]}$, 自由基加 成中间体可以发生 $\beta$-碳氧键断裂, 以水为唯一副产物, 实现羟基的脱除, 最后通过氢转移的方法淬灭烷基自由 基. 反应将可见光促进的 PCET 过程和 SCS 有机地结合 起来, 实现了醛/酮碳氧双键的断裂.

反应对于醛和酮具有较好的容忍性, 对于其它含氮 杂环如吡啶、苯并噻唑、哒嗪、喹啉等底物反应也能顺 利进行. 该方法可用于一系列含杂芳环结构的药物分子 或天然产物的后期烷基化修饰. 作者利用该方法合成了 甲吡酮、米力农并对氯雷他定及乙托贝特进行了烷基化 修饰. 最后, 结合机理实验和相关文献报道, 作者对反 应机制进行了合理推测. 综上所述, 该反应通过可见光 促进的 PCET 方式将羰基转化为碳自由基, 以羰基为烷 基自由基的直接来源, 是一种新的醛酮活化方式. 反应
具有原料易得、条件温和和底物适用性及官能团容忍性 良好等优点, 为含氮杂环化合物的修饰提供了一种新的 策略.

\section{References}

[1] Proctor. R. S. J.; Phipps, R. J. Angew. Chem., Int. Ed. 2019, 58, 13666.

[2] Carey, F. A.; Sundberg, R. J. Advanced Organic Chemistry: Part B: Reactions and Synthesis, Springer, Berlin, Heidelberg, 2001.

[3] Wang, H.; Dai, X.-J.; Li, C.-J. Nat. Chem. 2017, 9, 374.

[4] Chatgilialoglu, C.; Crich, D.; Komatsu, M.; Ryu, I. Chem. Rev. 1999, 99, 1991.

[5] Dong, J.-Y.; Wang, Z.; Wang, X.-C.; Song, H.-J.; Liu, Y.-X.; Wang, Q.-M. Sci. Adv. 2019, 5, EAAX9955.

[6] Tarantino, K. T.; Liu, P.; Knowles, R. R. J. Am. Chem. Soc. 2013, 135, 10022.

[7] Schönherr, H.; Cernak, T. Angew. Chem., Int. Ed. 2013, 52, 12256.

[8] Jin, J.; MacMillan, D. W. C. Nature 2015, 525, 87.

(Zhao, C.) 